\title{
GRB 050911: A BLACK HOLE-NEUTRON STAR MERGER OR A NAKED GRB
}

\author{
K. L. Page, ${ }^{1}$ A. R. King, ${ }^{1}$ A. J. Levan, ${ }^{2}$ P. T. O’Brien,,${ }^{1}$ J. P. Osborne, ${ }^{1}$ S. D. Barthelmy, ${ }^{3}$ A. P. Beardmore, ${ }^{1}$ \\ D. N. Burrows, ${ }^{4}$ S. Campana,${ }^{5}$ N. Gehrels, ${ }^{3}$ J. Graham, ${ }^{6}$ M. R. Goad, ${ }^{1}$ O. Godet, ${ }^{1}$ Y. Kaneko, ${ }^{7}$ \\ J. A. Kennea, ${ }^{4}$ C. B. Markwardt, ${ }^{3}$ D. E. Reichart ${ }^{8}$ T. Sakamoto, ${ }^{3}$ and N. R. Tanvir ${ }^{2}$ \\ Received 2005 November 11; accepted 2005 December 13; published 2006 January 10
}

\begin{abstract}
GRB 050911, discovered by the Swift Burst Alert Telescope, was not seen 4.6 hr later by the Swift X-ray Telescope, making it one of the very few X-ray nondetections of a gamma-ray burst (GRB) afterglow at early times. The $\gamma$-ray light curve shows at least three peaks, the first two of which $\left(\sim T_{0}-0.8\right.$ and $T_{0}+0.2 \mathrm{~s}$, where $T_{0}$ is the trigger time) were short, each lasting $0.5 \mathrm{~s}$. This was followed by later emission $10-20 \mathrm{~s}$ postburst. The upper limit on the unabsorbed X-ray flux was $1.7 \times 10^{-14} \mathrm{ergs} \mathrm{cm}^{-2} \mathrm{~s}^{-1}$ (integrating $46 \mathrm{ks}$ of data taken between September 11 and 18), indicating that the decay must have been rapid. All but one of the long bursts detected by Swift were above this limit at $\sim 4.6 \mathrm{hr}$, whereas the afterglows of short bursts became undetectable more rapidly. Deep observations with Gemini also revealed no optical afterglow $12 \mathrm{hr}$ after the burst, down to $r=$ 24.0 (5 $\sigma$ limit). We speculate that GRB 050911 may have been formed through a compact object (black holeneutron star) merger, with the later outbursts due to a longer disk lifetime linked to a large mass ratio between the merging objects. Alternatively, the burst may have occurred in a low-density environment, leading to a weak, or nonexistent, forward shock-the so-called "naked GRB" model.
\end{abstract}

Subject heading: gamma rays: bursts

\section{INTRODUCTION}

The bimodality in gamma-ray burst (GRB) durations has long been recognized (e.g., Kouveliotou et al. 1993) with the $90 \% \gamma$ ray emission interval $\left(T_{90}\right)$ peaking around 0.3 and $30 \mathrm{~s}$, with a minimum at $2 \mathrm{~s}$. The short-duration bursts also typically exhibit systematically harder emission than the longer ones (Kouveliotou et al. 1993). The revolution that transformed the study of longduration bursts via the identification of afterglows and host galaxies at cosmological redshifts has only just reached the short bursts. The recent discoveries of short-burst afterglows in several cases (e.g., GRB 050509B: Gehrels et al. 2005, Bloom et al. 2006; GRB 050709: Covino et al. 2005, Fox et al. 2005b, Hjorth et al. 2005a, Villasenor et al. 2005; GRB 050724: Barthelmy et al. 2005c; GRB 050813: D. B. Fox et al. 2006, in preparation) and the association of these with host galaxies of various morphological types (including elliptical galaxies) indicate that short GRBs have a different origin from the longer duration bursts. They are typically found at lower redshifts (e.g., Bloom et al. 2006; Berger et al. 2005; Tanvir et al. 2005) and have isotropic energies 3 orders of magnitude below those of the long GRBs. The definitive lack of detection of a supernova related to GRB 050509B (Hjorth et al. 2005b) supports the difference between long and short bursts. The observations to date are in line with what may be expected from GRBs occurring via compact object

\footnotetext{
${ }^{1}$ Department of Physics and Astronomy, University of Leicester, University Road, Leicester, LE1 7RH, UK; kpa@star.le.ac.uk.

${ }^{2}$ Centre for Astrophysics Research, University of Hertfordshire, Hatfield, AL10 9AB, UK.

${ }^{3}$ NASA Goddard Space Flight Center, Greenbelt, MD 20771.

${ }^{4}$ Department of Astronomy and Astrophysics, Pennsylvania State University, 525 Davey Laboratory, University Park, PA 16802.

${ }_{5}^{5}$ INAF, Osservatorio Astronomico di Brera, Via E. Bianchi 46, I-23807, Merate (LC), Italy.

${ }^{6}$ Space Telescope Science Institute, 3700 San Martin Drive, Baltimore, MD 21218.

${ }^{7}$ National Space Science and Technology Center, University of Alabama, Huntsville, AL 35805.

${ }^{8}$ Department of Physics and Astronomy, University of North Carolina at Chapel Hill, Chapel Hill, NC 27599.
}

mergers (neutron star-neutron star [NS-NS] or black holeneutron star [BH-NS]).

The two burst populations clearly overlap in the hardness duration parameter space, and it is interesting to ask what distinguishes the different classes for the cases where classification simply via $T_{90}$ is ambiguous. At least one of the short GRBs found by Swift-GRB 050724 (Barthelmy et al. 2005c)-has softer emission beyond the expected $T_{90}$ of $2 \mathrm{~s}$. In fact, these data showed that the distinction between long and short bursts is partly instrument-dependent. Using Swift data, the $\gamma$-ray light curve of that burst was found to consist of an initial hard spike (lasting about $0.25 \mathrm{~s}$ ), followed by another peak at $T_{0}+1.1 \mathrm{~s}$; this section of the light curve would have classified GRB 050724 as a short burst. However, faint, softer emission was detected by the Swift Burst Alert Telescope (BAT) out to $140 \mathrm{~s}$ after the trigger. Simulations showed that the Burst and Transient Source Experiment (BATSE) would not have detected this softer pulse at more than $0.3 \sigma$, obtaining $T_{90} \sim 0.43 \mathrm{~s}$. Similar behavior was seen for GRB 050709 (Fox et al. 2005b), and both Lazzati et al. (2001) and Connaughton (2002) investigated such "tails" in BATSE short-burst data. Norris \& Bonnell (2005) have looked at short bursts with extended emission, finding that they can be differentiated from long bursts by having spectral lags consistent with zero for their initial spike emission.

Huang et al. (2005) discuss GRB 040924. The duration of this burst $\left(T_{90} \sim 1.2 \mathrm{~s}\right)$ places it in the short category, but the authors conclude that it might belong at the short end of the long GRB distribution. The presence of a supernova signature in this burst strengthens their assertion (Soderberg et al. 2006). Likewise, GRB 000301C (Jensen et al. 2001) had $T_{90}=2.0 \mathrm{~s}$ but all the properties of a long-duration burst (e.g., star-forming host galaxy, optical and radio detections of the afterglow, and high redshift). These observations provide evidence that the long-duration population extends to at least 1-2 s.

GRB 050911 also appears to be a candidate for a burst whose classification via $T_{90}$ is unclear. Although $T_{90}$ places it in the long-burst category, any later X-ray or optical emission decayed rapidly and/or was extremely faint, characteristics more 
TABLE 1

SWIFT XRT ObSERving TIME LiNe FOR GRB 050911

\begin{tabular}{ccccr}
\hline \hline ObsID & Date & Start UT & Stop UT & $\begin{array}{c}\text { Exposure } \\
(\mathrm{s})\end{array}$ \\
\hline $00154630000 \ldots \ldots$ & 2005 Sep 11 & $20: 33: 00$ & $01: 08: 52$ & 6343 \\
$00154630001 \ldots \ldots$ & 2005 Sep 12 & $14: 16: 31$ & $20: 48: 30$ & 9954 \\
$00154630002 \ldots \ldots$ & 2005 Sep 14 & $01: 31: 14$ & $22: 43: 59$ & 13043 \\
$00154631003 \ldots \ldots$ & 2005 Sep 18 & $06: 33: 52$ & $22: 51: 57$ & 17137 \\
\hline
\end{tabular}

common for short bursts (see, e.g., Gehrels et al. 2005; D. B. Fox et al. 2006, in preparation). This Letter presents the Swift and ground-based observations of the burst ( $\S 2$ and 3 ), finding that it was only detected in $\gamma$-rays. Explanations for the lack of X-ray emission are explored in $\S 4$.

\section{SWIFT OBSERVATIONS}

The Swift Gamma-Ray Burst Explorer (Gehrels et al. 2004) is a multiwavelength mission designed to detect and study GRBs. The observatory consists of three instruments-the wide-field $\gamma$-ray BAT (Barthelmy et al. 2005b) and the two narrow-field instruments: the X-Ray Telescope (XRT; Burrows et al. 2005) and Ultraviolet/Optical Telescope (UVOT; Roming et al. 2005).

GRB 050911 was located by the BAT (trigger number 154630) on 2005 September 11 at 15:59:34 UT (Page et al. 2005b). The ground-calculated BAT position (Tueller et al. 2005) was R.A. $=00^{\mathrm{h}} 54^{\mathrm{m}} 52^{\mathrm{s}} 4$, decl. $=-38^{\circ} 51^{\prime} 42^{\prime \prime} .8(\mathrm{~J} 2000.0)$, with an uncertainty of 2.8 (radius, $90 \%$ containment). The value of $T_{90}$ was determined to be $\sim 16 \mathrm{~s}$, while $T_{50} \sim 13.7 \mathrm{~s}$; the $T_{90}$ fluence over $15-150 \mathrm{keV}$ is $\sim 3 \times 10^{-7} \mathrm{ergs} \mathrm{cm}^{-2}$, placing it at the lower end of the BAT distribution.

Due to a combination of the Earth-limb observing constraint and a temporary problem with the star trackers, the first XRT and UVOT observations of the position of GRB 050911 started $4.6 \mathrm{hr}$ after the burst trigger, when a $6.3 \mathrm{ks}$ exposure was obtained. Table 1 gives the details of this and the subsequent X-ray observations of the burst. Throughout this Letter, errors are given at the $90 \%$ significance level unless otherwise stated.

\section{1. $\gamma$-Ray Data}

Figure 1 plots the BAT light curve, showing two short spikes, each about $0.5 \mathrm{~s}$ in duration, close to $T_{0}-0.8$ and $T_{0}+0.2 \mathrm{~s}$ (where $T_{0}$ is the burst trigger time), followed by a slow rise and fall between $T_{0}+10$ and $T_{0}+20 \mathrm{~s}$.

The $T_{90}$ BAT spectrum can be well fitted over $15-150 \mathrm{keV}$ with a simple power law, of photon index $\Gamma=1.90_{-0.31}^{+0.33}$ $\left(\chi^{2} /\right.$ dof $\left.=54 / 56\right)$. Fitting either a Band function (Band et al. 1993) or a cutoff power law does not improve the fit.

Separate spectra for the time periods covering the first two spikes in the light curve $\left(T_{0}-4\right.$ to $\left.T_{0}+6 \mathrm{~s}\right)$ and the longer, broader peak $\left(T_{0}+6\right.$ to $\left.T_{0}+26 \mathrm{~s}\right)$ were extracted, to determine whether the later emission appeared softer, as was the case for the two short bursts GRB 050709 and GRB 050724 (Barthelmy et al. 2005c; Boer et al. 2005). Although there is some evidence for spectral softening with time $\left(\Gamma_{\text {early }}=1.82_{-0.67}^{+0.73} ; \Gamma_{\text {later }}=\right.$ $2.29_{-0.49}^{+1.23}$, within the errors the photon indices are consistent. However, as Barthelmy et al. (2005c) found for GRB 050724, simulations show it is likely that the later, weaker peak would not have been detected significantly by BATSE $(<1 \sigma)$; it should be noted, though, that the first peak would also have been marginal $(<3.5 \sigma)$, so BATSE may not have triggered on the event at all.

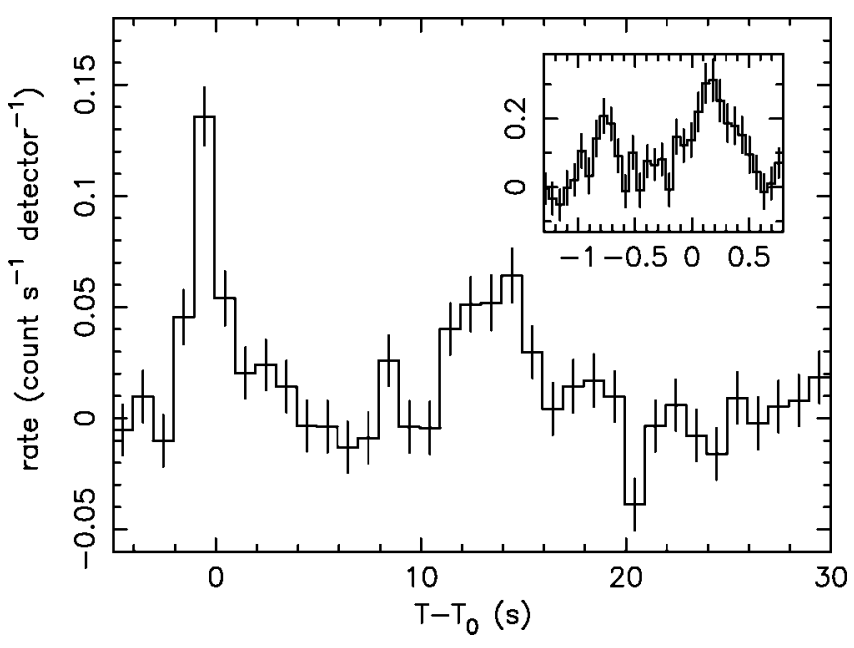

FIG. 1.-BAT light curve over $15-350 \mathrm{keV}$. The main plot shows both the initial short burst and the longer, later emission, while the inset zooms in on the two short peaks.

The hardness ratio $[S(50-100 \mathrm{keV}) /(25-50 \mathrm{keV})]$ is consistent with the ranges observed for both short and long bursts detected by BATSE and Swift. Following Norris \& Bonnell (2005), the spectral lag measurement for the initial peak is consistent with zero, as found for short bursts.

\subsection{X-Ray Data}

Only one source was detected in the BAT error circle by the XRT (Page et al. 2005c), but this was found to be constant over time and therefore was discounted as the afterglow of GRB 050911 (Page et al. 2005a).

For any other object in the BAT error circle, the $3 \sigma$ upper limit for the count rate is $4.0 \times 10^{-4}$ counts $\mathrm{s}^{-1}$ evaluated over the entire 46 ks exposure (see Table 1). The corresponding unabsorbed flux limit is $1.7 \times 10^{-14} \mathrm{ergs} \mathrm{cm}^{-2} \mathrm{~s}^{-1}$ over $0.3-$ $10 \mathrm{keV}$, when we assume a Crab-like spectrum with a Galactic absorbing column of $2.7 \times 10^{20} \mathrm{~cm}^{-2}$ (Dickey \& Lockman 1990). When we consider just the initial $6.3 \mathrm{ks}$ exposure at $4.6 \mathrm{hr}$ after the trigger, the upper limit is $1.25 \times 10^{-3}$ counts s$^{-1}$ (5.2 $\times 10^{-14} \mathrm{ergs} \mathrm{cm}^{-2} \mathrm{~s}^{-1}$, unabsorbed, over $\left.0.3-10 \mathrm{keV}\right)$.

Figure 2 shows the BAT light curve extrapolated into the $0.3-$ $10 \mathrm{keV}$ band (when we assume a slope of $\Gamma=1.9$ ) compared

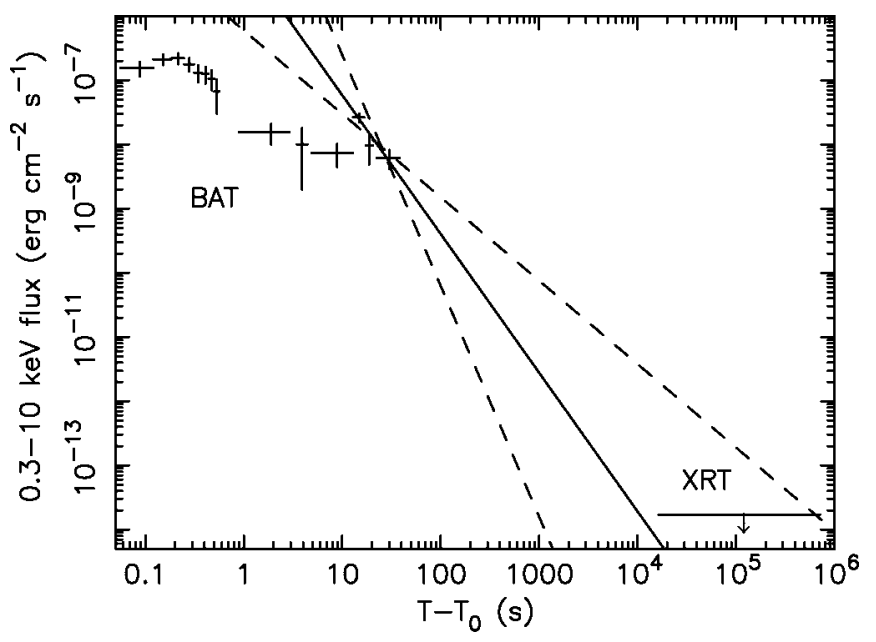

FIG. 2.-BAT flux light curve and XRT upper limit, showing an extrapolation of the power-law fit to the last peak in the BAT. 
to the XRT upper limit. Fitting a power-law decay to the later peak in the BAT light curve gives a slope of $\alpha=2.2_{-0.9}^{+1.4}$; the solid and dashed lines show $\alpha=2.2$ and the upper and lower limits, respectively. The X-ray upper limit is consistent with the extrapolation of the fit to the end of the BAT data.

\subsection{Optical and UV Data}

No new sources were found in the field by the UVOT, with $3 \sigma$ upper limits on the magnitude of between 20 and 21 for the optical and UV filters; the exposures were between 600 and $900 \mathrm{~s}$ in duration (Breeveld et al. 2005).

\section{GROUND-BASED OPTICAL OBSERVATIONS}

We observed the BAT position (Tueller et al. 2005) with the Gemini South Telescope and the Gemini Multi-Object Spectrograph (GMOS) instrument over two epochs (midpoint times of 2005 September 12 04:05:14 UT and 2005 September 15 05:33:18 UT). Images were obtained in the $r$ band, with exposure times of $1500 \mathrm{~s}(5 \times 300 \mathrm{~s})$ and $3000 \mathrm{~s}(10 \times 300 \mathrm{~s})$; $5^{\prime \prime}$ dithers were made between subsequent exposures to cover the chip gaps in the GMOS detectors, and the field of view of 5.5 covered the complete refined BAT error circle. Note that the conditions were suboptimal, with relatively poor seeing $\left(\sim 1^{\prime \prime} 2\right)$. No new sources were seen in the GMOS images in comparison to the Digitized Sky Survey; however, as the Gemini observations are significantly deeper, a further search for variability was conducted by performing a point-spread function matched image subtraction of the two epochs using the ISIS-II code of Alard \& Lupton (1998). To estimate the sensitivity to variable objects, a set of artificial stars was created in the first epoch image based on a zero point obtained from comparison to the USNO catalog, and the FWHM equal to that of stellar objects within the field, and the difference image investigated. Using this technique, a conservative $5 \sigma$ limiting magnitude of $r=24.0$ was determined for any variable object within the BAT error circle $12 \mathrm{hr}$ after the trigger.

No follow-up detections of GRB 050911 were reported through the GCN (Tristram et al. 2005; Castro-Tirado et al. 2005; Berger \& Boss 2005).

\section{DISCUSSION}

The nondetection of an X-ray afterglow is very unusual for Swift bursts. Before GRB 050911, only four other GRBs (050416B, 050502A, 050709, and 050509C) had been observed with the XRT with no afterglow detection; however, these were first observed with the XRT after $86 \mathrm{hr}, 11$ days, $39 \mathrm{hr}$, and 9 days, respectively, all significantly later than for GRB 050911. As of 2005 November 1, Swift slewed to GRBs 050603, $050714 \mathrm{~A}$, and 050827 with a greater delay than that for GRB 050911, yet X-ray afterglows were detected in these cases.

Swift bursts that were detected by the XRT before quickly fading below its sensitivity level (i.e., afterglows that would not have been located at $4.6 \mathrm{hr}$ ) were all short. (GRB 050421 is the exception to this rule and is discussed below.) GRB 050509B ( $T_{90} \sim 30 \mathrm{~ms}$; Hurkett et al. 2005; Barthelmy et al. 2005a; Gehrels et al. 2005) and GRB $050813\left(T_{90} \sim 0.6 \mathrm{~s}\right.$; Sato et al. 2005; D. B. Fox et al. 2006, in preparation) both showed no X-ray emission after a few thousand seconds. For GRB $050906\left(T_{90} \sim 128 \mathrm{~ms}\right.$; Parsons et al. 2005), only a possible, extremely faint X-ray counterpart was identified (Fox et al. 2005a). Likewise, GRB $050925\left(T_{90} \sim 72 \mathrm{~ms}\right.$; Holland et al. 2005; Beardmore et al. 2005) was not detected after a prompt slew, although there is a possibility that this BAT trigger was due to a new soft $\gamma$-ray repeater.

Comparison of our stringent early X-ray upper limit for GRB 050911 of $5.2 \times 10^{-14} \mathrm{ergs} \mathrm{cm}^{-2} \mathrm{~s}^{-1}$ with the light curves of other Swift bursts (e.g., Fig. 2 of Nousek et al. 2006) shows that the afterglow must have been an order of magnitude or more fainter than all of the other long bursts at $4.6 \mathrm{hr}$, with the possible exception of GRB 050421. The light curve of that burst (Godet et al. 2006) appears to be only the tail end of the prompt emission, with no evidence for an afterglow (probably due to the burst occurring in a low-density environment: a "naked" GRB).

The ratio between the $4.6 \mathrm{hr}$ XRT and $T_{90}$ BAT fluxes was measured for a large number of bursts and the lowest value found to be $\sim 4 \times 10^{-6}$. If we consider the GRB 050911 BAT flux $\left(1.86 \times 10^{-8} \mathrm{ergs} \mathrm{cm}^{-2} \mathrm{~s}^{-1}\right)$, this lowest observed ratio would predict an X-ray flux of $\sim 7 \times 10^{-14} \mathrm{ergs}^{-2} \mathrm{~s}^{-1}$ at $4.6 \mathrm{hr}$, higher than the upper limit determined. Thus, GRB 050911 was particularly X-ray faint for the measured $\gamma$-ray flux.

Our Gemini limit on the optical flux of GRB 050911 is among the deepest obtained for a long-duration Swift burst; only the afterglow of GRB 050412 was apparently fainter than this, with a limit of $R c=24.9$ only $2 \mathrm{hr}$ after the burst (Kosugi et al. 2005). However, optical afterglows for the recent short bursts GRB 050509B and GRB 050813 were undetected to deep limits via Keck, Gemini, and Very Large Telescope observations (e.g., Bloom et al. 2006; Hjorth et al. 2005b; Bloom 2005; Berger \& Gladders 2005). Deep observations of the handful of short bursts that were localized prior to Swift also failed to locate any afterglow candidates (e.g., Hurley et al. 2002; Klotz et al. 2003).

\subsection{Long or Short?}

From the discussion above, there are at least two possibilities for GRB 050911: either the light curve is similar to GRB 050421, showing no sign of emission due to a forward shock, or it is akin to those in the short-burst class. The calculated $T_{90}$ for GRB 050911 falls within the long-burst category, although the initial two peaks in the BAT light curve are both short ( 0.5 s each).

The BAT spectrum is not particularly hard. Short bursts tend to be spectroscopically harder than the long bursts; the spectral distinction not strong, though, and the photon index measured for GRB 050911 is within the range observed for both populations (e.g., Ghirlanda et al. 2004). However, any X-ray emission was unusually faint, more in keeping with measurements of short bursts (Fox et al. 2005b), which tend to fade below the XRT sensitivity within a few thousand seconds.

Both the short bursts GRB 050509B and GRB 050724 (Gehrels et al. 2005; Barthelmy et al. 2005a) were found to be associated with non-star-forming elliptical galaxies (thus, a compact binary merger origin is more likely than a hypernova). In the case of GRB 050911, the cluster EDCC 493 (Lumsden et al. 1992; $z=0.16$ ) is in the line of sight (Berger 2005), although without a more refined position than that from the BAT, it cannot be claimed with any certainty that the burst is associated with any of the galaxies within the cluster. EDCC 493 is not a rich cluster, and a rough estimate suggests that, with a radius of $\sim 0.2$, the chance of such a cluster intersecting the line of sight is a few percent, so the alignment may just be due to chance. No information about the star formation rate in this cluster is known.

If GRB 050911 is, as $T_{90}$ suggests, a long burst, then it is unusually X-ray faint. It could be due to a collapsar, with the 
progenitor initially in a binary system. If we assume the progenitor to be the secondary star in the system, it could receive a kick into a low-density environment when the primary star undergoes a supernova explosion. If a GRB forms with very little surrounding interstellar material, only a weak forward shock will occur and therefore little afterglow emission will result.

If, instead, GRB 050911 is interpreted as a compact object merger, the $\gamma$-ray emission out beyond $10 \mathrm{~s}$ has to be explained; as discussed earlier, the burst is not unique in this respect. Models involving two neutron stars are unlikely to produce the long and structured emission seen in GRB 050911, because the mass ratio in a NS-NS merger is inevitably close to unity. The disruption of both neutron stars occurs as a single event, and the light curve is controlled by the properties of the resulting neutron torus. However, if the mass ratio is far from unity, the disruption event can be more complex. Davies et al. (2005) consider a BH-NS merger with a mass ratio of $10: 1$. They find that mass transfer from the neutron star occurs in spurts, with instantaneous rates exceeding $100 M_{\odot} \mathrm{s}^{-1}$. Between these spurts, the neutron star remnant travels in a wider, eccentric orbit with an initial size dependent on how much of the angular momentum of the transferred mass is returned to the remnant. The orbits decay by gravitational radiation, causing subsequent bursts. For returned angular momentum fractions $\sim 0.5$, these intervals can be several seconds. If the black hole is formed relatively early on in this process, delayed accretion events could then cause later outbursts. When the neutron star remnant reaches a mass of $\sim 0.2 M_{\odot}$ it is completely disrupted, effectively terminating the accretion. Evidently more work is needed to model in detail the light curve predicted by this pattern of mass transfer, but the luminosities and timescales produced from a BH-NS merger resemble those in GRB 050911.

\section{CONCLUSIONS}

No X-ray afterglow emission was detected for GRB 050911, starting $4.6 \mathrm{hr}$ after the burst trigger. Comparison of the upper limit of the emission with other Swift-detected bursts (Nousek et al. 2006) demonstrates that any afterglow was at least an order of magnitude fainter than for any other long burst, with the possible exception of GRB 050421. The behavior could be due to either a BH-NS merger or a collapsar occurring in a region of low density, thus forming a naked GRB. Whatever mechanism produced GRB 050911, it was an unusual, X-raydark, burst.

The authors acknowledge support for this work at the University of Leicester by PPARC, at Pennsylvania State University by NASA, and in Italy by ASI. A. J. L. and N. R. T. acknowledge receipt of PPARC fellowships. We also thank the referee for useful comments and J. Norris for the spectral lag calculation.

\section{REFERENCES}

Alard, C., \& Lupton, R. H. 1998, ApJ, 503, 325

Band, D., et al. 1993, ApJ, 413, 281

Barthelmy, S., et al. 2005a, GCN Circ. 3385, http://gcn.gsfc.nasa.gov/gcn/gcn3/ 3385.gen 3

2005b, Space Sci. Rev., 120, 143

2005c, Nature, 438, 994

Beardmore, A. P., Page, K. L., Gehrels, N., Greiner, J., Kennea, J., Nousek, J., Osborne, J. P., \& Tagliaferri, G. 2005, GCN Circ. 4043, http://gcn.gsfc nasa.gov/gen/gen $3 / 4043 . g c n 3$

Berger, E. 2005, GCN Circ. 3962, http://gcn.gsfc.nasa.gov/gcn/gcn3/3962.gcn3

Berger, E., \& Boss, A. 2005, GCN Circ. 3966, http://gcn.gsfc.nasa.gov/gcn/ gcn3/3966.gcn3

Berger, E., \& Gladders, M. 2005, GCN Circ. 3806, http://gcn.gsfc.nasa.gov/ $\mathrm{gcn} / \mathrm{gcn} 3 / 3806 . \mathrm{gcn} 3$

Berger, E., et al. 2005, Nature, 438, 988

Bloom, J. S. 2005, GCN Circ. 3802, http://gcn.gsfc.nasa.gov/gcn/gcn3/3802.gcn3

Bloom, J. S., et al. 2006, ApJ, in press (astro-ph/0505480)

Boer, M., et al. 2005, GCN Circ. 3653, http://gcn.gsfc.nasa.gov/gcn/gcn3/ 3653.gcn 3

Breeveld, A. A., Page, K., Poole, T. S., Marshall, F., Chester, M., Mészáros, P., \& Nousek, J. 2005, GCN Circ. 3969, http://gcn.gsfc.nasa.gov/gcn/gcn3/ 3969.gcn3

Burrows, D. N., et al. 2005, Space Sci. Rev., 120, 165

Castro-Tirado, A. J., de Ugarte Postigo, A., Guziy, S., Jelinek, M., Gorosabel, J., Tristram, P., \& Yock, Ph. 2005, GCN Circ. 3968, http://gcn.gsfc.nasa.gov/ $\mathrm{gcn} / \mathrm{gcn} 3 / 3968 . \mathrm{gcn} 3$

Connaughton, V. 2002, ApJ, 567, 1028

Covino, S., et al. 2005, A\&A, submitted (astro-ph/0509144)

Davies, M. B., Levan, A. J., \& King, A. R. 2005, MNRAS, 356, 54

Dickey, J. M., \& Lockman, F. J. 1990, ARA\&A, 28, 215

Fox, D. B., Pagani, C., Angelini, L., Burrows, D. N., Osborne, J. P., \& La Parola, V. 2005a, GCN Circ. 3956, http://gcn.gsfc.nasa.gov/gcn/gcn3/3956.gcn3

Fox, D. B., et al. 2005b, Nature, 437, 845

Gehrels, N., et al. 2004, ApJ, 611, 1005

- 2005, Nature, 437, 851

Ghirlanda, G., Ghisellini, G., \& Celotti, A. 2004, A\&A, 422, L55

Godet, O., et al. 2006, A\&A, in press

Hjorth, J., et al. 2005a, Nature, 437, 859
Hjorth, J., et al. 2005b, ApJ, 630, L117

Holland, S. T., et al. 2005, GCN Circ. 4034, http://gcn.gsfc.nasa.gov/gcn/gcn3/ 4034.gcn3

Huang, K. Y., et al. 2005, ApJ, 628, L93

Hurkett, C., et al. 2005, GCN Circ. 3381, http://gcn.gsfc.nasa.gov/gcn/gcn3/ 3381.gen 3

Hurley, K., et al. 2002, ApJ, 567, 447

Jensen, B. L., et al. 2001, A\&A, 370, 909

Klotz, A., Boer, M., \& Ateia, J.-L. 2003, A\&A, 404, 815

Kosugi, G., Kawai, N., Aoki, K., Hattori, T., Ohta, K., \& Yamada, T. 2005, GCN Circ. 3263, http://gcn.gsfc.nasa.gov/gen/gen3/3263.gen3

Kouveliotou, C., Meegan, C. A., Fishman, G. J., Bhat, N. P., Briggs, M. S., Koshut, T. M., Paciesas, W. S., \& Pendleton, G. N. 1993, ApJ, 413, L101

Lazzati, D., Ramirez-Ruiz, E., \& Ghisellini, G. 2001, A\&A, 379, L39

Lumsden, S. L., Nichol, R. C., Collins, C. A., \& Guzzo, L. 1992, MNRAS, 258,1

Norris, J. P., \& Bonnell, J. T. 2005, ApJ, submitted

Nousek, J. A., et al. 2006, ApJ, in press (astro-ph/0508332)

Page, K., Burrows, D., Gehrels, N., O'Brien, P., Marshall, F., \& Boyd, P. 2005a, GCN Circ. 3976, http://gcn.gsfc.nasa.gov/gen/gcn3/3976.gcn3

Page, K., Chester, M., Cummings, J., Gehrels, N., Kennea, J., Markwardt, C., \& Palmer, D. 2005b, GCN Circ. 3961, http://gcn.gsfc.nasa.gov/gcn/gcn3/ 3961.gen3

Page, K., Kennea, J., \& Burrows, D. 2005c, GCN Circ. 3967, http://gcn.gsfc .nasa.gov/gen/gen3/3967.gen3

Parsons, A., et al. 2005, GCN Circ. 3935, http://gcn.gsfc.nasa.gov/gcn/gcn3/ 3935.gcn 3

Roming, P. W. A., et al. 2005, Space Sci. Rev., 120, 95

Sato, G., et al. 2005, GCN Circ. 3793, http://gen.gsfc.nasa.gov/gen/gen3/ 3793.gen 3

Soderberg, A. M., et al. 2006, ApJ, 636, 391

Tanvir, N., Chapman, R., Levan, A., \& Priddey, R. 2005, Nature, 438, 991

Tristram, P., Castro-Tirado, A. J., Guziy, S., de Ugarte Postigo, A., Jelinek, M., Gorosabel, J., \& Yock, Ph. 2005, GCN Circ. 3965, http://gcn.gsfc.nasa .gov/gcn/gcn $3 / 3965$.gcn 3

Tueller, J., et al. 2005, GCN Circ. 3964, http://gcn.gsfc.nasa.gov/gcn/gcn3/ 3964.gen3

Villasenor, J. S., et al. 2005, Nature, submitted (astro-ph/0510190) 